\title{
On an Infinite Product for the Ratio of Consecutive Prime Numbers Edigles Guedes ${ }^{1}$ and Prof. Dr. K. Raja Rama Gandhi ${ }^{2}$ Number Theorist, Brazil ${ }^{1}$ and \\ Resource person in Math for Oxford University Press and Professor in Math at BITS-Vizag ${ }^{2}$
}

ABSTRACT. The main objective of this paper is to develop an infinite product formula for the ratio of consecutive prime numbers, using Jacobi elliptic functions.

\section{INTRODUCTION}

The Rosser's theorem [2] states that $p_{n}$ is $\operatorname{larger}$ than $n \log n$. This can be improved by the following pair of bounds:

$$
\log n+\log \log n-1<\frac{p_{n}}{n}<\log n+\log \log n
$$

for $n>6$.

\section{THEOREM}

THEOREM 1. For $n>9$, we have

$$
\begin{aligned}
& \frac{p_{n}}{p_{n+1}}+\theta_{n}=\frac{e^{539 \pi / 1024}}{1+e^{-336875 \pi / 16}} \times \frac{1+e^{-10779461 \pi / 512}}{1+e^{-5389461 \pi / 256}} \times \frac{1+e^{-10778383 \pi / 512}}{1+e^{-2694461 \pi / 128}} \times \frac{1+e^{-10777305 \pi / 512}}{1+e^{-5388383 \pi / 256}} \\
& \times \frac{1+e^{-10776227 \pi / 512}}{1+e^{-1346961 \pi / 64}} \times \frac{1+e^{-10775149 \pi / 512}}{1+e^{-5387305 \pi / 256}} \times \frac{9988}{9987} \times \frac{1+e^{-5929 \pi / 512}}{1+e^{-3773 \pi / 256}} \times \frac{1+e^{-4851 \pi / 512}}{1+e^{-1617 \pi / 128}} \\
& \times \frac{1+e^{-3773 \pi / 512}}{1+e^{-2695 \pi / 256}} \times \frac{1+e^{-2695 \pi / 512}}{1+e^{-539 \pi / 64}} \times \frac{1+e^{-1617 \pi / 512}}{1+e^{-1617 \pi / 256}} \times \frac{1+e^{-539 \pi / 512}}{1+e^{-539 \pi / 128}} \times \frac{1}{1+e^{-539 \pi / 256}} \\
& \times e^{-\frac{\pi}{2}\left(\frac{35}{32}+\frac{1}{4 n}+\frac{1}{8 n^{2}}-\frac{49 n^{2}}{128(n+1)^{2}}-\frac{21 n^{4}}{512(n+1)^{4}}\right)} \prod_{l=1}^{\infty}\left[\frac{1+e^{-2 l \pi\left(\frac{35}{32}+\frac{1}{4 n}+\frac{1}{8 n^{2}}-\frac{49 n^{2}}{128(n+1)^{2}}-\frac{21 n^{4}}{512(n+1)^{4}}\right)}}{1+e^{-(2 l-1) \pi\left(\frac{35}{32}+\frac{1}{4 n}+\frac{1}{8 n^{2}}-\frac{49 n^{2}}{128(n+1)^{2}}-\frac{21 n^{4}}{512(n+1)^{4}}\right)}}\right],
\end{aligned}
$$

where

$$
\theta_{n}<0.153
$$

and $p_{n}$ is the $n$-th prime number.

Proof. Firstly, we consider the sequence of prime numbers

$$
2<3<5<7<11<13<17<19<\cdots p_{n-2}<p_{n-1}<p_{n}<p_{n+1} .
$$

Second, we note that

$$
\begin{aligned}
0<\frac{2}{3}<1,0<\frac{3}{5}<1,0 & <\frac{5}{7}<1,0<\frac{7}{11}<1,0<\frac{11}{13}<1,0<\frac{13}{17}<1,0<\frac{17}{19}<1, \ldots, \\
0 & <\frac{p_{n-2}}{p_{n-1}}<1,0<\frac{p_{n-1}}{p_{n}}<1,0<\frac{p_{n}}{p_{n+1}}<1 .
\end{aligned}
$$

Then, we define that

$$
k:=k_{n, n+1}=\frac{p_{n}}{p_{n+1}}
$$

where $k_{n, n+1}$ is the $k$ modulus. 
In $[2$, p. 83], we knew that

$$
k^{1 / 2}=\frac{\theta_{2}}{\theta_{3}}=\frac{\theta_{2}(0)}{\theta_{3}(0)}=\frac{\theta_{2}(0 \mid \tau)}{\theta_{3}(0 \mid \tau)}
$$

where $\tau$ is the parameter and $\theta_{2}(z \mid \tau)$ and $\theta_{3}(z \mid \tau)$ are Jacobi theta functions.

On the other hand, in [2, p. 85], we have

$$
\theta_{2}=2 q^{1 / 4} G \prod_{l=1}^{\infty}\left(1+q^{2 l}\right)^{2}
$$

and

$$
\theta_{3}=G \prod_{l=1}^{\infty}\left(1+q^{2 l-1}\right)^{2}
$$

So, we obtain

$$
k^{1 / 2}=\frac{\theta_{2}}{\theta_{3}}=\frac{2 q^{1 / 4} G \prod_{l=1}^{\infty}\left(1+q^{2 l}\right)^{2}}{G \prod_{l=1}^{\infty}\left(1+q^{2 l-1}\right)^{2}}=2 q^{1 / 4} \prod_{l=1}^{\infty}\left(\frac{1+q^{2 l}}{1+q^{2 l-1}}\right)^{2},
$$

multiplying by $k^{1 / 2}$ in both members of (8), we have

$$
k=4 q^{1 / 2} \prod_{l=1}^{\infty}\left(\frac{1+q^{2 l}}{1+q^{2 l-1}}\right)^{4}
$$

In [3], we encounter

$$
q(k)=e^{\pi i \tau}=e^{-\pi K^{\prime}(k) / K(k)}=e^{-\pi K\left(\sqrt{1-k^{2}}\right) / K(k)} .
$$

We knew that [4] $K(k)$ can be expressed as a power series

$$
K(k)=\frac{\pi}{2} \sum_{l=0}^{\infty}\left[\frac{(2 l) !}{2^{2 l}(l !)^{2}}\right]^{2} k^{2 l}=\frac{\pi}{2} \sum_{l=0}^{\infty}\left[\frac{(2 l-1) ! !}{(2 l) ! !}\right]^{2} k^{2 l} .
$$

It can be expressed by asymptotic expansion

$$
K(k) \approx \frac{\pi}{2}+\frac{\pi}{8} \frac{k^{2}}{1-k^{2}}-\frac{\pi}{16} \frac{k^{4}}{1-k^{2}}
$$

If we substitute (12) in (10), then we find

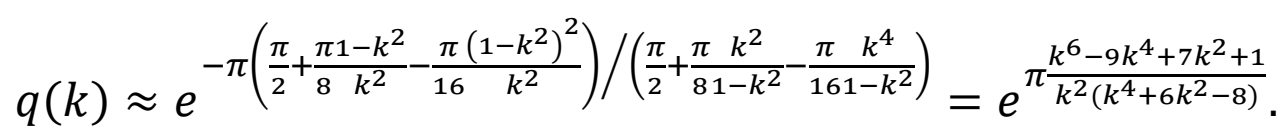

We calculate the asymptotic expansion of $\frac{k^{6}-9 k^{4}+7 k^{2}+1}{k^{2}\left(k^{4}+6 k^{2}-8\right)}$, that is,

$$
\frac{k^{6}-9 k^{4}+7 k^{2}+1}{k^{2}\left(k^{4}+6 k^{2}-8\right)}=-\frac{1}{8 k^{2}}-\frac{31}{32}+\frac{49 k^{2}}{128}+\frac{21 k^{4}}{512}+O\left(k^{5}\right)=-\left[\frac{1}{8 k^{2}}+\frac{31}{32}-\frac{49 k^{2}}{128}-\frac{21 k^{4}}{512}-O\left(k^{5}\right)\right]
$$

We take (14) in (13)

$$
q(k) \approx e^{-\pi\left(\frac{1}{8 k^{2}}+\frac{31}{32}-\frac{49 k^{2}}{128}-\frac{21 k^{4}}{512}\right)}
$$


Substituting (15) in (9), it follows that

$$
k \approx 4 e^{-\frac{\pi}{2}\left(\frac{1}{8 k^{2}}+\frac{31}{32}-\frac{49 k^{2}}{128}-\frac{21 k^{4}}{512}\right)} \prod_{l=1}^{\infty}\left[\frac{1+e^{-2 l \pi\left(\frac{1}{8 k^{2}}+\frac{31}{32}-\frac{49 k^{2}}{128}-\frac{21 k^{4}}{512}\right)}}{1+e^{-(2 l-1) \pi\left(\frac{1}{8 k^{2}}+\frac{31}{32}-\frac{49 k^{2}}{128}-\frac{21 k^{4}}{512}\right)}}\right]^{4} .
$$

We put (4) in (16)

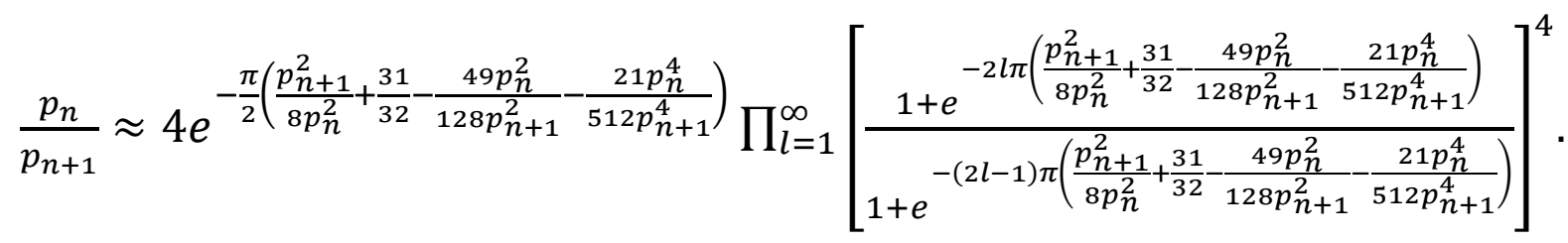

Using (1), we discover that

$$
\begin{gathered}
\frac{(n+1)^{2}[\log (n+1)+\log \log (n+1)]^{2}}{8 n^{2}(\log n+\log \log n-1)^{2}}+\frac{31}{32}-\frac{49 n^{2}(\log n+\log \log n)^{2}}{128(n+1)^{2}[\log (n+1)+\log \log (n+1)-1]^{4}} \\
-\frac{21 n^{4}(\log n+\log \log n)^{4}}{512(n+1)^{4}[\log (n+1)+\log \log (n+1)-1]^{4}} \\
<\frac{p_{n+1}^{2}}{8 p_{n}^{2}}+\frac{31}{32}-\frac{49 p_{n}^{2}}{128 p_{n+1}^{2}}-\frac{21 p_{n}^{4}}{512 p_{n+1}^{4}} \\
<\frac{(n+1)^{2}[\log (n+1)+\log \log (n+1)]^{2}}{8 n^{2}(\log n+\log \log n-1)^{2}}+\frac{31}{32}-\frac{49 n^{2}(\log n+\log \log n-1)^{2}}{128(n+1)^{2}[\log (n+1)+\log \log (n+1)]^{2}} \\
-\frac{21 n^{4}(\log n+\log \log n-1)^{4}}{512(n+1)^{4}[\log (n+1)+\log \log (n+1)]^{4}}
\end{gathered}
$$

whereas factors involving logarithms tend to 1 , we have the approximation

$$
\begin{gathered}
\frac{p_{n+1}^{2}}{8 p_{n}^{2}}+\frac{31}{32}-\frac{49 p_{n}^{2}}{128 p_{n+1}^{2}}-\frac{21 p_{n}^{4}}{512 p_{n+1}^{4}} \sim \frac{(n+1)^{2}}{8 n^{2}}+\frac{31}{32}-\frac{49 n^{2}}{128(n+1)^{2}}-\frac{21 n^{4}}{512(n+1)^{4}} \\
=\frac{n^{2}+2 n+1}{8 n^{2}}+\frac{31}{32}-\frac{49 n^{2}}{128(n+1)^{2}}-\frac{21 n^{4}}{512(n+1)^{4}} \\
=\frac{1}{8}+\frac{31}{32}+\frac{1}{4 n}+\frac{1}{8 n^{2}}-\frac{49 n^{2}}{128(n+1)^{2}}-\frac{21 n^{4}}{512(n+1)^{4}} \\
=\frac{35}{32}+\frac{1}{4 n}+\frac{1}{8 n^{2}}-\frac{49 n^{2}}{128(n+1)^{2}}-\frac{21 n^{4}}{512(n+1)^{4}} .
\end{gathered}
$$

We substitute (18) in (17)

$$
\frac{p_{n}}{p_{n+1}} \sim 4 e^{-\frac{\pi}{2}\left(\frac{35}{32}+\frac{1}{4 n}+\frac{1}{8 n^{2}}-\frac{49 n^{2}}{128(n+1)^{2}}-\frac{21 n^{4}}{512(n+1)^{4}}\right)} \prod_{l=1}^{\infty}\left[\frac{1+e^{-2 l \pi\left(\frac{35}{32}+\frac{1}{4 n}+\frac{1}{8 n^{2}}-\frac{49 n^{2}}{128(n+1)^{2}}-\frac{21 n^{4}}{512(n+1)^{4}}\right)}}{1+e^{-(2 l-1) \pi\left(\frac{35}{32}+\frac{1}{4 n}+\frac{1}{8 n^{2}}-\frac{49 n^{2}}{128(n+1)^{2}}-\frac{21 n^{4}}{512(n+1)^{4}}\right)}}\right]^{4} .
$$

Ergo, we can consider that

$$
\frac{p_{n}}{p_{n+1}}=4 G_{n} e^{-\frac{\pi}{2}\left(\frac{35}{32}+\frac{1}{4 n}+\frac{1}{8 n^{2}}-\frac{49 n^{2}}{128(n+1)^{2}}-\frac{21 n^{4}}{512(n+1)^{4}}\right)} \prod_{l=1}^{\infty}\left[\frac{1+e^{-2 l \pi\left(\frac{35}{32}+\frac{1}{4 n}+\frac{1}{8 n^{2}}-\frac{49 n^{2}}{128(n+1)^{2}}-\frac{21 n^{4}}{512(n+1)^{4}}\right)}}{1+e^{-(2 l-1) \pi\left(\frac{35}{32}+\frac{1}{4 n}+\frac{1}{8 n^{2}}-\frac{49 n^{2}}{128(n+1)^{2}}-\frac{21 n^{4}}{512(n+1)^{4}}\right)}}\right]^{4} .
$$


In other words,

$$
G_{n}=\frac{p_{n}}{4 p_{n+1} e^{-\frac{\pi}{2}\left(\frac{35}{32}+\frac{1}{4 n}+\frac{1}{8 n^{2}}-\frac{49 n^{2}}{128(n+1)^{2}}-\frac{21 n^{4}}{512(n+1)^{4}}\right)} \prod_{l=1}^{\infty}\left[\frac{1+e^{-2 l \pi\left(\frac{35}{32}+\frac{1}{4 n}+\frac{1}{8 n^{2}}-\frac{49 n^{2}}{128(n+1)^{2}}-\frac{21 n^{4}}{512(n+1)^{4}}\right)}}{1+e^{-(2 l-1) \pi\left(\frac{35}{32}+\frac{1}{4 n}+\frac{1}{8 n^{2}}-\frac{49 n^{2}}{128(n+1)^{2}}-\frac{21 n^{4}}{512(n+1)^{4}}\right)}}\right]^{4}}
$$

On the other hand, we suppose that

$$
G_{n}=\alpha_{n} G,
$$

where

$$
G=\lim _{n \rightarrow \infty} \frac{p_{n}}{4 p_{n+1} e^{-\frac{\pi}{2}\left(\frac{35}{32}+\frac{1}{4 n}+\frac{1}{8 n^{2}}-\frac{49 n^{2}}{128(n+1)^{2}}-\frac{21 n^{4}}{512(n+1)^{4}}\right)} \prod_{l=1}^{\infty}\left[\frac{1+e^{-2 l \pi\left(\frac{35}{32}+\frac{1}{4 n}+\frac{1}{8 n^{2}}-\frac{49 n^{2}}{128(n+1)^{2}}-\frac{21 n^{4}}{512(n+1)^{4}}\right)}}{1+e^{-(2 l-1) \pi\left(\frac{35}{32}+\frac{1}{4 n}+\frac{1}{8 n^{2}}-\frac{49 n^{2}}{128(n+1)^{2}}-\frac{21 n^{4}}{512(n+1)^{4}}\right)}}\right]^{4}}
$$

By Rosser's theorem (1), we put

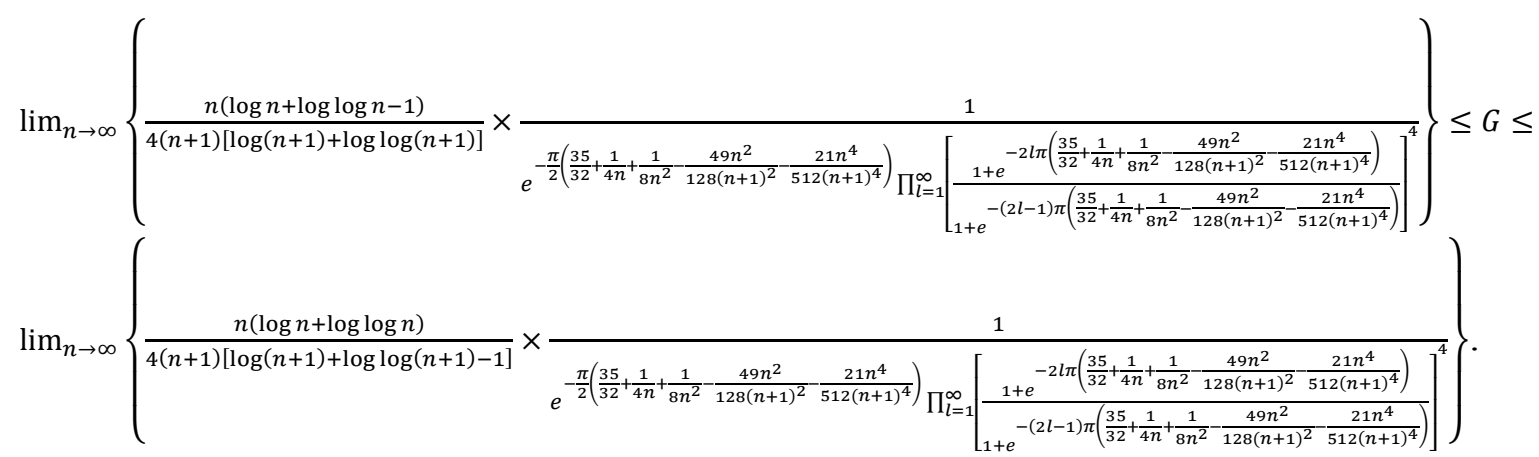

We calculate the limit in both members for obtain of

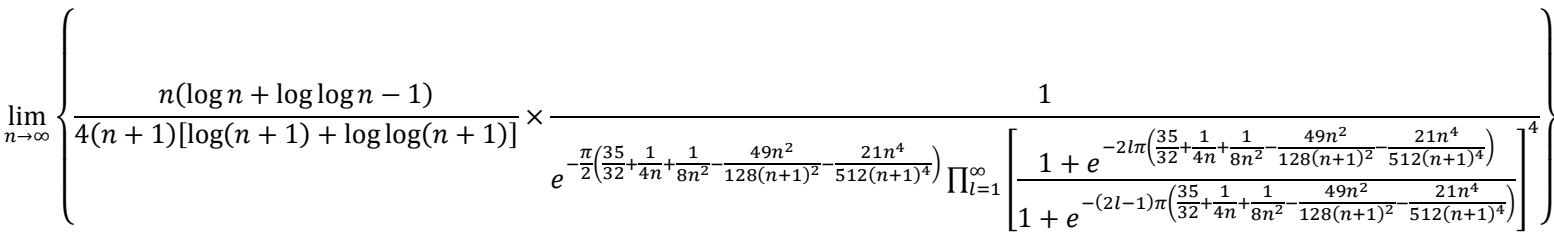

$$
\begin{aligned}
& \approx \frac{1}{4} \times \frac{e^{539 \pi / 1024}}{1+e^{-336875 \pi / 16}} \times \frac{1+e^{-10779461 \pi / 512}}{1+e^{-5389461 \pi / 256}} \times \frac{1+e^{-10778383 \pi / 512}}{1+e^{-2694461 \pi / 128}} \times \frac{1+e^{-10777305 \pi / 512}}{1+e^{-5388383 \pi / 256}} \\
& \times \frac{1+e^{-10776227 \pi / 512}}{1+e^{-1346961 \pi / 64}} \times \frac{1+e^{-10775149 \pi / 512}}{1+e^{-5387305 \pi / 256}} \times \frac{9989}{9987} \times \frac{1+e^{-5929 \pi / 512}}{1+e^{-3773 \pi / 256}} \times \frac{1+e^{-4851 \pi / 512}}{1+e^{-1617 \pi / 128}} \\
& \times \frac{1+e^{-3773 \pi / 512}}{1+e^{-2695 \pi / 256}} \times \frac{1+e^{-2695 \pi / 512}}{1+e^{-539 \pi / 64}} \times \frac{1+e^{-1617 \pi / 512}}{1+e^{-1617 \pi / 256}} \times \frac{1+e^{-539 \pi / 512}}{1+e^{-539 \pi / 128}} \times \frac{1}{1+e^{-539 \pi / 256}}
\end{aligned}
$$

and

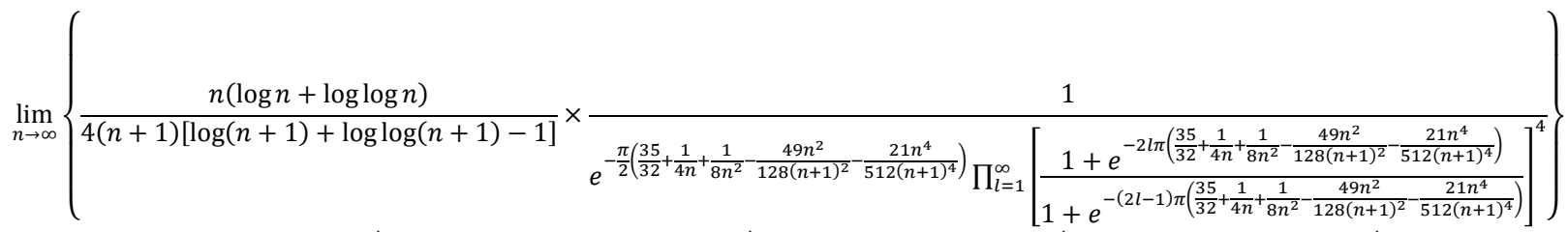

$$
\begin{aligned}
& \approx \frac{1}{4} \times \frac{e^{539 \pi / 1024}}{1+e^{-336875 \pi / 16}} \times \frac{1+e^{-10779461 \pi / 512}}{1+e^{-5389461 \pi / 256}} \times \frac{1+e^{-10778383 \pi / 512}}{1+e^{-2694461 \pi / 128}} \times \frac{1+e^{-10777305 \pi / 512}}{1+e^{-5388383 \pi / 256}} \\
& \times \frac{1+e^{-10776227 \pi / 512}}{1+e^{-1346961 \pi / 64}} \times \frac{1+e^{-10775149 \pi / 512}}{1+e^{-5387305 \pi / 256}} \times \frac{9988}{9987} \times \frac{1+e^{-5929 \pi / 512}}{1+e^{-3773 \pi / 256}} \times \frac{1+e^{-4851 \pi / 512}}{1+e^{-1617 \pi / 128}} \\
& \times \frac{1+e^{-3773 \pi / 512}}{1+e^{-2695 \pi / 256}} \times \frac{1+e^{-2695 \pi / 512}}{1+e^{-539 \pi / 64}} \times \frac{1+e^{-1617 \pi / 512}}{1+e^{-1617 \pi / 256}} \times \frac{1+e^{-539 \pi / 512}}{1+e^{-539 \pi / 128}} \times \frac{1}{1+e^{-539 \pi / 256}},
\end{aligned}
$$


hence, we can assume that

$$
\begin{gathered}
G \approx \frac{1}{4} \times \frac{e^{539 \pi / 1024}}{1+e^{-336875 \pi / 16}} \times \frac{1+e^{-10779461 \pi / 512}}{1+e^{-5389461 \pi / 256}} \times \frac{1+e^{-10778383 \pi / 512}}{1+e^{-2694461 \pi / 128}} \\
\times \frac{1+e^{-10777305 \pi / 512}}{1+e^{-5388383 \pi / 256}} \times \frac{1+e^{-10776227 \pi / 512}}{1+e^{-1346961 \pi / 64}} \times \frac{1+e^{-10775149 \pi / 512}}{1+e^{-5387305 \pi / 256}} \times \frac{9988}{9987} \\
\times \frac{1+e^{-5929 \pi / 512}}{1+e^{-3773 \pi / 256}} \times \frac{1+e^{-4851 \pi / 512}}{1+e^{-1617 \pi / 128}} \times \frac{1+e^{-3773 \pi / 512}}{1+e^{-2695 \pi / 256}} \times \frac{1+e^{-2695 \pi / 512}}{1+e^{-539 \pi / 64}} \\
\times \frac{1+e^{-1617 \pi / 512}}{1+e^{-1617 \pi / 256}} \times \frac{1+e^{-539 \pi / 512}}{1+e^{-539 \pi / 128}} \times \frac{1}{1+e^{-539 \pi / 256}} .
\end{gathered}
$$

From (22), (21), (20) and (19), it follows that

$\frac{p_{n}}{p_{n+1}}=$

$$
\begin{aligned}
& \alpha_{n} \times \frac{e^{539 \pi / 1024}}{1+e^{-336875 \pi / 16}} \times \frac{1+e^{-10779461 \pi / 512}}{1+e^{-5389461 \pi / 256}} \times \frac{1+e^{-10778383 \pi / 512}}{1+e^{-2694461 \pi / 128}} \times \frac{1+e^{-10777305 \pi / 512}}{1+e^{-5388383 \pi / 256}} \\
& \times \frac{1+e^{-10776227 \pi / 512}}{1+e^{-1346961 \pi / 64}} \times \frac{1+e^{-10775149 \pi / 512}}{1+e^{-5387305 \pi / 256}} \times \frac{9988}{9987} \times \frac{1+e^{-5929 \pi / 512}}{1+e^{-3773 \pi / 256}} \times \frac{1+e^{-4851 \pi / 512}}{1+e^{-1617 \pi / 128}} \\
& \times \frac{1+e^{-3773 \pi / 512}}{1+e^{-2695 \pi / 256}} \times \frac{1+e^{-2695 \pi / 512}}{1+e^{-539 \pi / 64}} \times \frac{1+e^{-1617 \pi / 512}}{1+e^{-1617 \pi / 256}} \times \frac{1+e^{-539 \pi / 512}}{1+e^{-539 \pi / 128}} \times \frac{1}{1+e^{-539 \pi / 256}} \\
& \times e^{-\frac{\pi}{2}\left(\frac{35}{32}+\frac{1}{4 n}+\frac{1}{8 n^{2}}-\frac{49 n^{2}}{128(n+1)^{2}}-\frac{21 n^{4}}{512(n+1)^{4}}\right)} \prod_{l=1}^{\infty}\left[\frac{1+e^{-2 l \pi\left(\frac{35}{32}+\frac{1}{4 n}+\frac{1}{8 n^{2}}-\frac{49 n^{2}}{128(n+1)^{2}}-\frac{21 n^{4}}{512(n+1)^{4}}\right)}}{\left.1+e^{-(2 l-1) \pi\left(\frac{35}{32}+\frac{1}{\left.4 n+\frac{1}{8 n^{2}}-\frac{49 n^{2}}{128(n+1)^{2}}-\frac{21 n^{4}}{512(n+1)^{4}}\right)}\right.}\right],}\right.
\end{aligned}
$$

in other words, we conclude that

$$
\begin{aligned}
\frac{p_{n}}{p_{n+1}} & +\theta_{n}=\frac{e^{539 \pi / 1024}}{1+e^{-336875 \pi / 16}} \times \frac{1+e^{-10779461 \pi / 512}}{1+e^{-5389461 \pi / 256}} \times \frac{1+e^{-10778383 \pi / 512}}{1+e^{-2694461 \pi / 128}} \times \frac{1+e^{-10777305 \pi / 512}}{1+e^{-5388383 \pi / 256}} \\
& \times \frac{1+e^{-10776227 \pi / 512}}{1+e^{-1346961 \pi / 64}} \times \frac{1+e^{-10775149 \pi / 512}}{1+e^{-5387305 \pi / 256}} \times \frac{9988}{9987} \times \frac{1+e^{-5929 \pi / 512}}{1+e^{-3773 \pi / 256}} \times \frac{1+e^{-4851 \pi / 512}}{1+e^{-1617 \pi / 128}} \\
& \times \frac{1+e^{-3773 \pi / 512}}{1+e^{-2695 \pi / 256}} \times \frac{1+e^{-2695 \pi / 512}}{1+e^{-539 \pi / 64}} \times \frac{1+e^{-1617 \pi / 512}}{1+e^{-1617 \pi / 256}} \times \frac{1+e^{-539 \pi / 512}}{1+e^{-539 \pi / 128}} \times \frac{1}{1+e^{-539 \pi / 256}} \\
& \times e^{-\frac{\pi}{2}\left(\frac{35}{32}+\frac{1}{4 n}+\frac{1}{8 n^{2}}-\frac{49 n^{2}}{128(n+1)^{2}}-\frac{21 n^{4}}{512(n+1)^{4}}\right)} \prod_{l=1}^{\infty}\left[\frac{1+e^{-2 l \pi\left(\frac{35}{32}+\frac{1}{4 n}+\frac{1}{8 n^{2}}-\frac{49 n^{2}}{128(n+1)^{2}}-\frac{21 n^{4}}{512(n+1)^{4}}\right)}}{1+e^{-(2 l-1) \pi\left(\frac{35}{32}+\frac{1}{4 n}+\frac{1}{8 n^{2}}-\frac{49 n^{2}}{128(n+1)^{2}}-\frac{21 n^{4}}{512(n+1)^{4}}\right)}}\right],
\end{aligned}
$$

where

$$
\theta_{n}<0.153
$$

for $n>9$, see table 1 . 
Table 1. In this table, we have: first column: $n$; second column: $p_{n}$; third column: $p_{n+1}$; fourth column: $\frac{p_{n}}{p_{n+1}}$; fifth column: the fractional part of $\frac{p_{n}}{p_{n+1}}$; sixth column: $\psi_{n}^{*}$; seventh column: $\psi_{n}^{*}-\frac{p_{n}}{p_{n+1}}$.

\begin{tabular}{|c|c|c|c|c|c|c|}
\hline 10 & 29 & 31 & $\frac{29}{31}$ & 0.935483870968 & 0.98897721869 & 0.0534933477223 \\
\hline 1 & 31 & 37 & $\frac{31}{37}$ & 0.837837837838 & 0.990490778545 & 0.152652940706 \\
\hline 12 & 37 & 41 & $\frac{37}{41}$ & 0.90243902439 & 0.991680519818 & 0.089241495428 \\
\hline 13 & 41 & 43 & $\overline{43}$ & 0.953488372093 & 0.992635412886 & 0.0391470407925 \\
\hline 4 & 43 & 47 & $\frac{43}{47}$ & 0.914893617021 & 0.993415470002 & 0.0785218529805 \\
\hline 15 & 47 & 53 & $\frac{47}{53}$ & 0.88679245283 & 0.994062430525 & 0.107269977694 \\
\hline 16 & 53 & 59 & $\frac{53}{59}$ & 0.898305084746 & 0.994606098095 & 0.0963010133487 \\
\hline 17 & 59 & 61 & $\frac{59}{61}$ & 0.967213114754 & 0.995068244543 & 0.027855129789 \\
\hline 18 & 61 & 67 & $\frac{61}{67}$ & 0.910447761194 & 0.995465095077 & 0.0850173338824 \\
\hline 19 & 67 & 71 & $\frac{67}{71}$ & 0.943661971831 & 0.995808956326 & 0.0521469844949 \\
\hline 20 & 71 & 73 & $\begin{array}{l}\overline{73} \\
73\end{array}$ & 0.972602739726 & 0.996109310172 & 0.0235065704456 \\
\hline 1 & 73 & 79 & $\begin{array}{l}\frac{15}{79} \\
79\end{array}$ & 0.924050632912 & 0.996373565309 & 0.0723229323973 \\
\hline 22 & 79 & 83 & $\frac{73}{83}$ & 0.951807228916 & 0.996607584107 & 0.0448003551913 \\
\hline 23 & 83 & 89 & $\overline{89}$ & 0.932584269663 & 0.996816058669 & 0.0642317890063 \\
\hline 2 & 89 & 97 & $\overline{97}$ & 0.917525773196 & 0.997002783666 & 0.0794770104697 \\
\hline 25 & 97 & 101 & $\frac{97}{101}$ & 0.960396039604 & 0.997170857231 & 0.0367748176272 \\
\hline 26 & 101 & 103 & $\begin{array}{l}\frac{101}{103} \\
103\end{array}$ & 0.980582524272 & 0.997322830911 & 0.0167403066389 \\
\hline 27 & 103 & 107 & $\overline{107}$ & 0.96261682243 & 0.997460822969 & 0.0348440005384 \\
\hline 28 & 107 & 109 & $\frac{7}{9}$ & 0.981651376147 & 0.997586604994 & 0.0159352288466 \\
\hline 29 & 109 & 113 & $\begin{array}{l}\frac{109}{113} \\
113\end{array}$ & 0.964601769912 & 0.9977016688 & 0.0330998988881 \\
\hline 30 & 113 & 127 & $\frac{26}{27}$ & 0.889763779528 & 0.997807278603 & 0.108043499075 \\
\hline
\end{tabular}


Table 2. In this table, we have: first column: $n$; second column: $p_{n}$; third column: $p_{n+1}$; fourth column: $\frac{p_{n}}{p_{n+1}}$; fifth column: the fractional part of $\frac{p_{n}}{p_{n+1}}$; sixth column: $\psi_{n}^{*}$; seventh column: $\psi_{n}^{*}-\frac{p_{n}}{p_{n+1}}$.

$\begin{array}{ccccccc}10 & 29 & 31 & \frac{29}{31} & 0.93548387 & 0.98897711 & 0.05349324 \\ 100 & 541 & 547 & \frac{541}{547} & 0.98903107 & 0.99957136 & 0.010540289 \\ 1000 & 7919 & 7927 & \frac{7919}{7927} & 0.99899079 & 1.0000534 & 0.0010626618 \\ 10000 & 104729 & 104743 & \frac{104729}{104743} & 0.99986633 & 1.0000954 & 0.00022909612 \\ 100000 & 1299709 & 1299721 & \frac{1299709}{1299721} & 0.99999076 & 1.000099 & 0.00010880395\end{array}$

Remark:

$$
\begin{aligned}
& \psi_{n}^{*}=\frac{e^{539 \pi / 1024}}{1+e^{-336875 \pi / 16}} \times \frac{1+e^{-10779461 \pi / 512}}{1+e^{-5389461 \pi / 256}} \times \frac{1+e^{-10778383 \pi / 512}}{1+e^{-2694461 \pi / 128}} \times \frac{1+e^{-10777305 \pi / 512}}{1+e^{-5388383 \pi / 256}} \\
& \times \frac{1+e^{-10776227 \pi / 512}}{1+e^{-1346961 \pi / 64}} \times \frac{1+e^{-10775149 \pi / 512}}{1+e^{-5387305 \pi / 256}} \times \frac{9988}{9987} \times \frac{1+e^{-5929 \pi / 512}}{1+e^{-3773 \pi / 256}} \times \frac{1+e^{-4851 \pi / 512}}{1+e^{-1617 \pi / 128}} \\
& \times \frac{1+e^{-3773 \pi / 512}}{1+e^{-2695 \pi / 256}} \times \frac{1+e^{-2695 \pi / 512}}{1+e^{-539 \pi / 64}} \times \frac{1+e^{-1617 \pi / 512}}{1+e^{-1617 \pi / 256}} \times \frac{1+e^{-539 \pi / 512}}{1+e^{-539 \pi / 128}} \times \frac{1}{1+e^{-539 \pi / 256}} \\
& \times e^{-\frac{\pi}{2}\left(\frac{35}{32}+\frac{1}{4 n}+\frac{1}{8 n^{2}}-\frac{49 n^{2}}{128(n+1)^{2}}-\frac{21 n^{4}}{512(n+1)^{4}}\right)} \prod_{l=1}^{\infty}\left[\frac{1+e^{-2 l \pi\left(\frac{35}{32}+\frac{1}{4 n}+\frac{1}{8 n^{2}}-\frac{49 n^{2}}{128(n+1)^{2}}-\frac{21 n^{4}}{512(n+1)^{4}}\right)}}{1+e^{-(2 l-1) \pi\left(\frac{35}{32}+\frac{1}{4 n}+\frac{1}{8 n^{2}}-\frac{49 n^{2}}{128(n+1)^{2}}-\frac{21 n^{4}}{512(n+1)^{4}}\right)}}\right]
\end{aligned}
$$

\section{REFERENCES}

[1] http://en.wikipedia.org/wiki/Prime_number_theorem, available in April 22, 2013.

[2] Armitage, J. V. and Eberlein, W. F., Elliptic Functions, London Mathematical Society, 2006.

[3] http://functions.wolfram.com/EllipticFunctions/EllipticNomeQ/, available in November 3, 2013.

[4] http://en.wikipedia.org/wiki/Elliptic_integral, available in November 3, 2013. 